\title{
STEM and Elemental Analysis by EDS and EELS for Two-dimensional Atomic Structure Containing $\mathrm{Au}$ and $\mathrm{Cu}$
}

Hidetaka Sawada ${ }^{1,2,3^{*}}$, Konstantin B. Borisenko ${ }^{1}$, Ichiro Ohnishi ${ }^{3}$, Yu Jimbo ${ }^{3}$, Eiji Okunishi ${ }^{3}$, and Angus I. Kirkland ${ }^{1,2}$

1. Department of Materials, University of Oxford, Parks Road, Oxford, UK.

2. Electron Physical Sciences Imaging Centre (ePSIC), Diamond Light Source Ltd, Didcot, Oxford, UK.

3. JEOL Ltd, 3-1-2 Musashino, Akishima, Tokyo, Japan.

* Corresponding author: hsawada@jeol.co.jp

In high-technology engineering of semiconducting devices, $\mathrm{Au}$ is frequently used for fine wiring due to its high electrical conductivity and chemical inertness. As integration of circuits has become higher and size of electronic devices has become smaller according to Moore's law, it is essentially important that nanometer-sized Au shows chemical and structural stability under normal operating temperatures and atmospheric conditions, to avoid potential degradation of device performance. In this study, we show that nanometer-sized $\mathrm{Au}$ is corroded in air in the presence of $\mathrm{Cu}$ at room temperature during ageing to form a compound of $\mathrm{Au}$. The compound consists of a belt-like two-dimensional material, containing $\mathrm{Au}$ and $\mathrm{Cu}[1]$.

$\mathrm{Cu}$ is vacuum deposited as pretreatment of $\mathrm{W}$ filaments to leave a small amount of residual $\mathrm{Cu}$ on the $\mathrm{W}$ wire. This filament was then used to deposit a few milligram of $\mathrm{Au}$ on a $\mathrm{C}$-film coated microscope grid. During deposition of $\mathrm{Au}$, residual $\mathrm{Cu}$ also evaporated. The film was then stored in air at room temperature for about 100 days to further aging. The aged film observed by scanning transmission electron microscopy (STEM) showed a belt-like structure with large lattice spacing around Au particles.

Figure 1 shows a high-resolution annular dark field (ADF) STEM dark field image of the aged film. The microscope we used was a $300 \mathrm{kV}$ aberration corrected microscope (JEOL, JEM-ARM300F) equipped with cold field emission gun. The 5-15-nm-sized particles visible in bright (white) contrast are Au. Around the Au particles, there are belt-like structures indicated by yellow arrows. We denote these as pedestrian crossing structures (PCS) hereinafter. These have shorter widths (approximately 2-5 nm) and longer lengths (approximately 5-20 nm). The spacing between the basal planes in the structure (perpendicular to the direction of longer axis of these particles) was determined to be $0.5 \pm 0.01 \mathrm{~nm}$. Electron energy loss spectroscopy (EELS) revealed that the rows of atomic columns in the PCS consist primarily of Au. Figure 2(a) shows a STEM ADF image of the PCS and Figs. 2 (b) - (d) show EDS elemental maps from a PCS with several gold particles. EDS analysis using dual silicon drift detectors (SDD) was performed with a probe current of $60 \mathrm{pA}$ using a convergent semi-angle of $30 \mathrm{mrad}$. The strong white contrast in Fig. 2(a) and 2(e) and rhombic particle with stripes are the gold particles and the PCS. To grasp the locations of these particles, we added yellow circles for gold particles and a light green rectangle to raw elemental maps of Figs. 2 (b) - (d) as shown in Figs. 2 (e) - (h). Figs. 2(c) and $2(\mathrm{~g})$ show elemental maps of $\mathrm{Cu}$, indicating that $\mathrm{Cu}$ was contained in both of $\mathrm{Au}$ particles and the PCS. Fig. 2 (i) shows an EDS X-ray spectrum from the region of the PCS. Although O signal was detected in this spectrum, the map of $\mathrm{O}$ shows mostly uniform intensity over the carbon film as shown in Figs. 2(d) and 2(h).

References: 
[1] H Sawada et al., submitted.

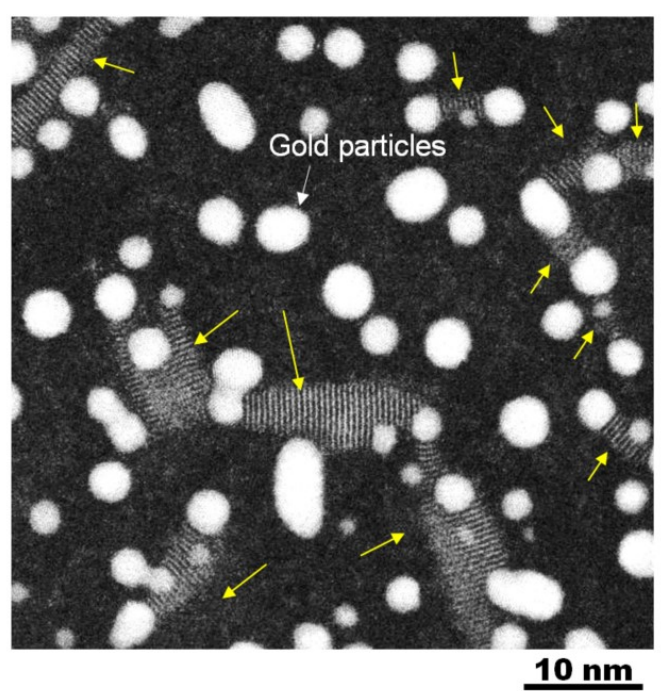

Figure 1. High-resolution ADF STEM image of a PCS with Au particles at $300 \mathrm{kV}$, which are indicated by yellow arrows.
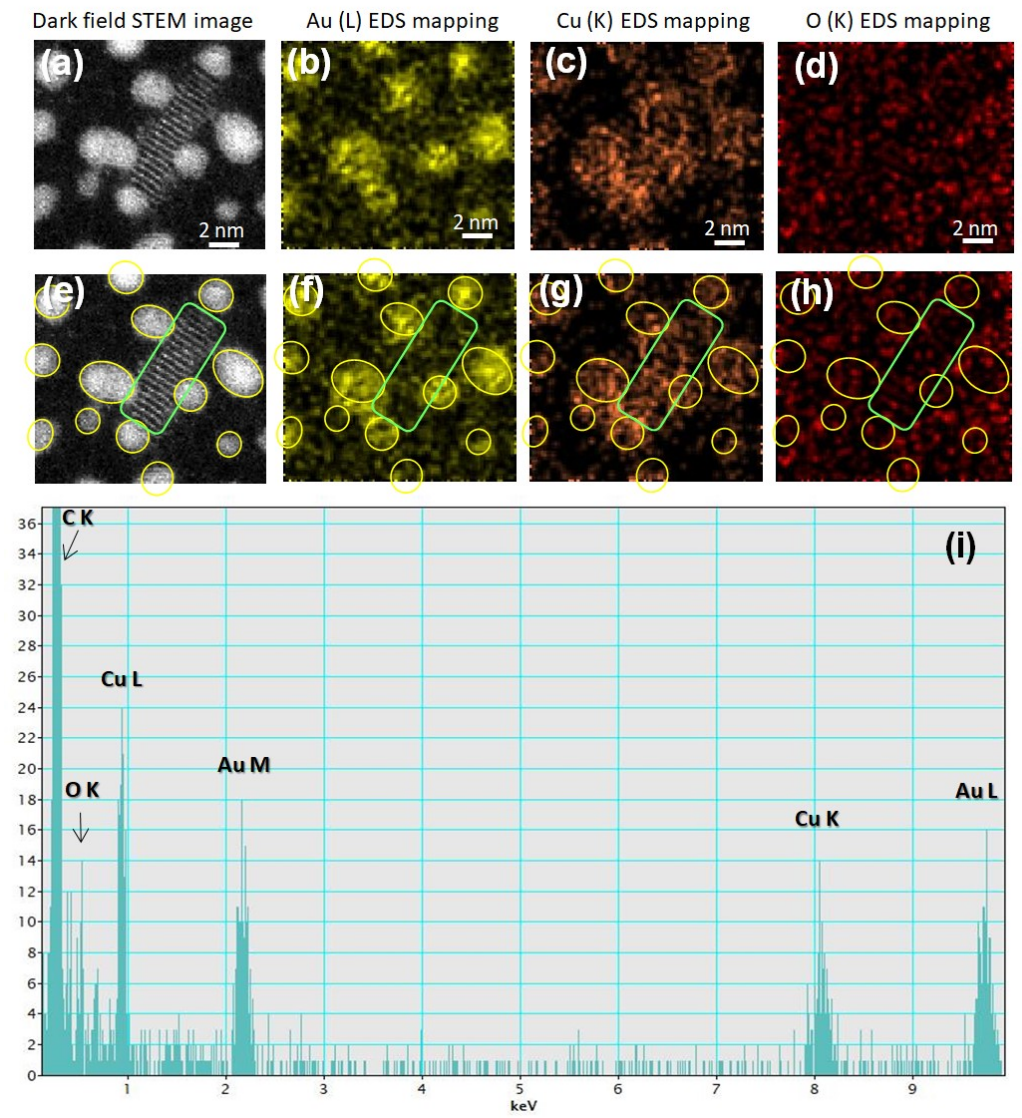

Figure 2. STEM ADF image of a PCS (pedestrian crossing structure) (a),(e) and simultaneously obtained EDS elemental maps of $\mathrm{Au}(\mathrm{b}, \mathrm{f}), \mathrm{Cu}(\mathrm{c}, \mathrm{g})$, and $\mathrm{O}(\mathrm{d}, \mathrm{h})$ with 100 times averaging with pixel dwell time of $0.5 \mathrm{~ms}$ at $80 \mathrm{kV}$. The size of these elemental maps are $84 \mathrm{x} 75$ pixel $(0.17 \mathrm{~nm} / \mathrm{pixel})$ (i) Xray spectrum taken from area of the PCS, which is indicated by green rectangle. Sample particles were made on the carbon film on gold grid. $\mathrm{C}$ was also detected from the carbon film. 